EPJ Web of Conferences 95,01011 (2015)

DOI: $10.1051 /$ epjconf/20159501011

C) Owned by the authors, published by EDP Sciences, 2015

\title{
Delta isobars in neutron stars
}

\author{
Giuseppe Pagliara ${ }^{1}$, a , Alessandro Drago ${ }^{1}$, Andrea Lavagno², and Daniele Pigato ${ }^{2}$ \\ ${ }^{1}$ Dip. di Fisica e Scienze della Terra dell'Università di Ferrara and \\ INFN Sez. di Ferrara, Via Saragat 1, I-44100 Ferrara, Italy \\ ${ }^{2}$ Department of Applied Science and Technology, Politecnico di Torino, Italy and \\ Istituto Nazionale di Fisica Nucleare (INFN), Sezione di Torino, Italy
}

\begin{abstract}
The appearance of delta isobars in beta-stable matter is regulated by the behaviour of the symmetry energy at densities larger than saturation density. We show that by taking into account recent constraints on the density derivative of the symmetry energy and the theoretical and experimental results on the excitations of delta isobars in nuclei, delta isobars are necessary ingredients for the equations of state used for studying neutron stars. We analyze the effect of the appearance of deltas on the structure of neutron stars: as in the case of hyperons, matter containing delta is too soft for allowing the existence of $2 M_{\odot}$ neutron stars. Quark stars on the other hand, could reach very massive configurations and they could form from a process of conversion of hadronic stars in which an initial seed of strangeness appears through hyperons.
\end{abstract}

\section{Introduction}

Understanding the properties of strongly interacting matter at densities of a few times saturation density, which are likely to be reached in the core of neutron stars, is one of the most interesting and challenging problem in nuclear and hadron physics. The difficulties arise basically because of the impossibility to solve QCD at those densities neither by perturbative approaches nor by lattice techniques. Many alternative strategies are available in the literature: microscopic calculations based on nucleon-nucleon interactions [1], phenomenological relativistic mean field models whose parameters are tuned at nuclear matter saturation [2] and more recently quantum Monte Carlo simulations and chiral effective field theories [3-5]. The matter inside a neutron star, besides being compressed to large densities by gravity, is also very neutron reach due to beta-equilibrium. This gives the opportunity to study at the same time both the isospin dependence of the equation of state (referring in particular to the symmetry energy) and its composition at large densities (referring to the possible appearance of baryons heavier than the nucleon).

None of the above mentioned calculation techniques has a real predictive power for what concerns the properties of stellar matter: only observations and measurements can finally put strong constraints on the equation of state. In the last years, due to the remarkable improvements of pulsar timing techniques, it has been discovered that the "canonical" value of the mass, $1.4 M_{\odot}$, is far below the maximum mass which must be of at least $2 M_{\odot}[6,7]$ (with an error of only a few percent). This constraint alone allows to rule out some of the proposed equations of state or to substantially reduce the

\footnotetext{
ae-mail: pagliara@fe.infn.it
} 


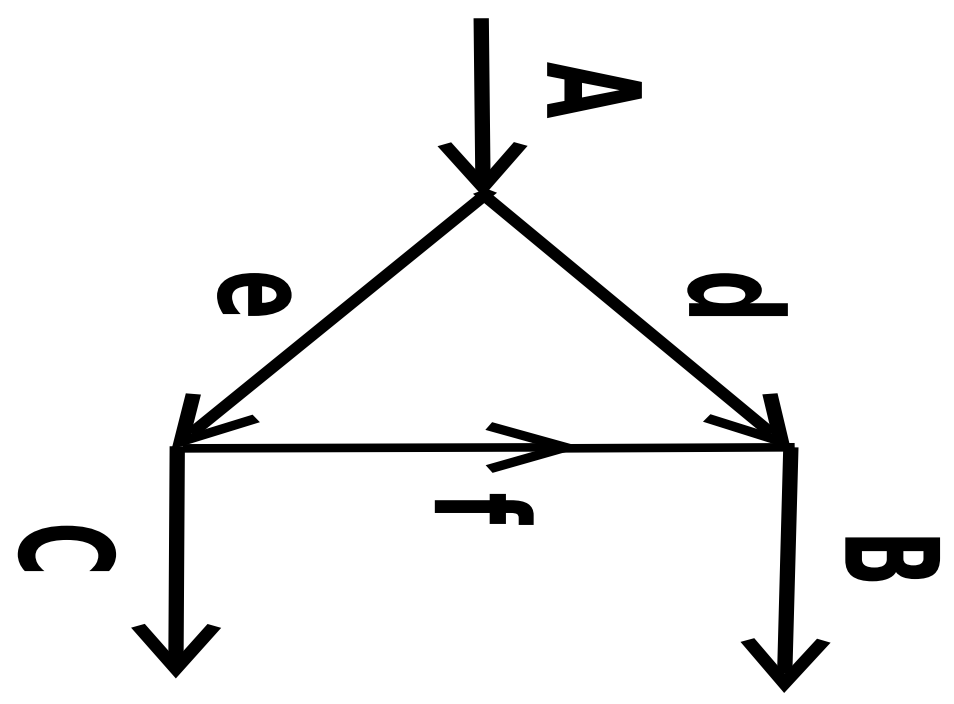

Figure 1. Couplings(ratios) of deltas with $\sigma$ and $\omega$ mesons. The shaded area indicates the range of values suggested by the analyses of scattering data, see [22].

phase space of the parameters of the models. Considering hyperons for instance, while in relativistic mean field models the free parameters can be tuned to fulfill the observational limit (see for instance [8]), in more realistic calculations based in microscopic nucleon-nucleon interactions the appearance of hyperons is accompanied by a strong softening of the equation of state which leads to maximum masses much below $2 M_{\odot}$ [9-11]: the so called "hyperon puzzle" stems from the difficulties in fulfilling the astrophysical limits and, at the same time, to account for the formation of hyperons which, being only slightly heavier than the nucleons, should form at large densities. A possible way out is that the nucleonic equation of state is so stiff that even for the $2 M_{\odot}$ star the central density is below the threshold for the appearance of hyperons: a possible example has been given in [12] where at $3.5 n_{0}$ (where $n_{0}$ is the saturation density) a mass of $2.09 M_{\odot}$ is obtained and hyperons are not yet formed (for a specific choice of the $\Lambda N N$ potential). If larger masses will be measured in the future, one possible candidate being the $2.4 M_{\odot}$ of Ref. [13], this kind of solution probably could not work anymore and the hyperon puzzle would be strengthened.

Measurements of neutron stars radii would be extremely useful for constraining the equation of state but it is clearly very hard to precise measure the radius of an object with a size of the order of $10 \mathrm{~km}$ and which is kpc far from us. Indeed all measurements of radii are affected by systematic uncertainties concerning the modeling of the surface temperature of the star and its atmosphere. Observations of quiescent low-mass X-ray binaries for instance lead to conclude that stars with radii of the order of $9.5 \mathrm{~km}$ exist in Ref.[14, 15]. A different analysis of the same stellar objects provided values close to $12 \mathrm{~km}$ [16]. Similarly, a radius larger than $11.1 \mathrm{~km}$ has been inferred in [17] for the millisecond pulsar PSRJ0437-4715, assuming that its mass is of $1.76 M_{\odot}$. While large radii, $R \sim 14 \mathrm{~km}$ for the canonical mass would imply a stiff equation of state and therefore small central densities, small radii, $R \sim 11 \mathrm{~km}$, imply a soft equation of state and therefore a large central density (see for instance [18]). Clearly a precise measurement of a radius would be important to clarify whether the formation of hyperons really represents a puzzle for the physics of neutron stars. 


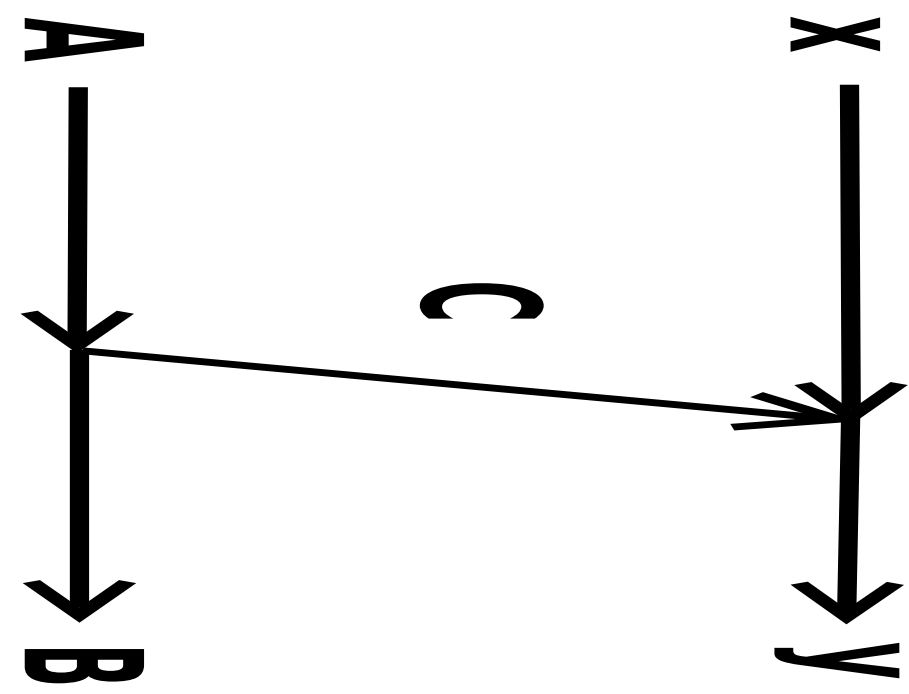

Figure 2. Thresholds densities of $\Delta^{-}$and $\Lambda$ as functions of $L$ for two choices of the coupling $x_{\omega \Delta}$.

Besides hyperons, also delta isobars have a mass enough close to the nucleon mass that they could possibly form in the center of neutron stars causing a softening of the equation of state. As found in Refs.[19, 20] within the framework of quantum hadrodynamics, the formation of deltas in beta-stable matter is regulated by the strength of the coupling of the baryons with the $\rho$ meson which in turn is connected with the symmetry energy and its density dependence. The recent experimental constraints on the symmetry energy and its density derivative, the $L$ parameter [21], suggested to reconsider the role of delta isobars in neutron stars in Ref. [22]. We will review in the following the main results concerning the formation of delta isobars in neutron stars and we will discuss how to reconcile the precise measurements of high masses and the hints of small radii within a scenario of two coexisting families of compact stars.

\section{Equation of state for $\Delta$ isobars}

We work within a relativistic mean field model parametrization recently proposed in Ref.[23]. When including deltas, three free parameters must be fixed which correspond to the ratios of the couplings of the deltas and the nucleons with the mesons: $x_{\sigma \Delta}, x_{\omega \Delta}, x_{\rho \Delta}$. The most natural choice, given the flavor composition of deltas, is the universal coupling choice which consists in assuming $x_{\sigma \Delta}=x_{\omega \Delta}=x_{\rho \Delta}=$ 1. There have been many theoretical and experimental studies on the excitations of delta resonances in nuclear matter by means of electron and pion scattering and photoabsorption processes [24-30]. A general conclusion one can draw from these studies is that these particles feel, in the nuclear medium, a non-relativistic binding potential larger than the the nucleon's one, of the order of $30 \mathrm{MeV}$ larger. When working within a relativistic mean field model, as in [24], this translates into values of $x_{\omega \Delta}$ 10-20\% smaller than $x_{\sigma \Delta}$. We display in Fig.1, the constraints on the values of these couplings. Unfortunately, we do not have any information on $x_{\rho \Delta}$ that will be therefore fixed to 1 .

With this setup one can now study the thresholds for the appearance of deltas in beta-stable matter. In Ref. [19], for the case of universal couplings, it has been shown that deltas would appear at densities much too high to be reached in the core of neutron stars. As shown in Ref.[22], this result is basically 
related to the large value of $L$ which in the Glendenning's parametrizations is of the order of $80 \mathrm{MeV}$ thus larger than the recent estimates of Ref. [21] where the maximum allowed value of $L$ is of 62 $\mathrm{MeV}$. Only by fixing the couplings with the $\rho$ meson to zero, deltas could appear but then the value of the symmetry energy would be too low [19]. By use of a simple toy-model one can easily show the correlation between $L$ and the thresholds of deltas: we use the parametrization GM3 [31] but we add a density dependence in the coupling to the $\rho$ meson. A typical form factor for such a density dependent coupling is given by $g_{\rho}(n)=g_{\rho}\left(n_{0}\right) e^{-a\left(n / n_{0}-1\right)}$ [32]. By varying $a$ it is possible to vary $L$ and to study then how the thresholds of deltas and hyperons are modified. Results are shown in Fig.2 for $\Delta^{-}$and $\Lambda$ which are the most relevant heavy baryons in beta stable matter. The continuous lines correspond to the case of universal couplings while the dashed lines to the case in which $x_{\omega \Delta}$ is suppressed with respect to $x_{\sigma \Delta}$ as indicated by the scattering data. In the most unfavorable case for deltas, i.e. $x_{\omega \Delta}=1$ and $L$ equal to the maximum allowed value, the deltas would appear at a density of $\sim 0.45 \mathrm{fm}^{-3}$ only slightly larger than the density for the formation of $\Lambda$. In the most favorable case, i.e. $x_{\omega \Delta}=0.9$ and $L$ equal to the minimum allowed value, the delta would appear at a density close to $2 n_{0}$ while the $\Lambda$ at a density of $\sim 0.55 \mathrm{fm}^{-3}$. Clearly in all cases, deltas form in beta-stable matter at densities which are easily reached in neutron stars. In the case of the equation of state that will be adopted in the following, the SFHo parametrization of [23], $L \sim 47 \mathrm{MeV}$, and indeed deltas appear before hyperons as shown in [22]. Including these additional degrees of freedom in the equations of state is therefore necessary when modeling neutron stars and in turn this could lead to a "delta isobars puzzle" similar to the "hyperon puzzle".

\section{Properties of compact stars}

For the following discussion we fix the couplings $x_{\sigma \Delta}=x_{\rho \Delta}=1$ and ${ }_{\omega \Delta}=0.9$ and for the hyperons we use the same couplings as in [33]. In Fig.3 we show the corresponding mass radius relations (both the gravitational and the baryonic mass). The softening caused by the appearance of new baryons leads to a maximum mass, in this case, of about $1.6 M_{\odot}$ thus non compatible with the $2 M_{\odot}$ limit. Even artificially excluding hyperons from the equation of state, as found in [22], does not solve the problem because the appearance of deltas is already enough to reduce the maximum mass below the observed value. It is interesting to notice that the stars with masses between 1.4 and $1.6 M_{\odot}$ have radii within $10.5-11.5 \mathrm{~km}$ thus compatible with the small radii inferred in [15], see also [34]. The question is clearly: what type of matter composes the high masses stars and how are they formed? Quark stars represent a viable, although highly uncertain possibility: perturbative QCD calculations have indeed shown that those stellar objects could have masses up to $2.75 M_{\odot}$ [35-39]. We display in Fig.3 an example, QS1, taken from [40]. Quark star matter is very rich in strangeness, indeed almost 1/3 of this matter is strange, and therefore to form this matter from hadronic matter it is necessary to have an initial seed of strangeness. Hyperons, when they start to form in the center of hadronic stars, constitute the way strange quark matter can be nucleated as extensively studied in previous works on quantum and thermal nucleation [41-46]. A shown in Fig.3 stars containing hyperons can then convert into quark stars (see the green arrows). In the left panel of the figure, the red point labels the hadronic star configuration, in particular its baryonic mass and radius, for which hyperons start to form in the center of the star. Taking the same baryonic mass on the quark stars branch, the blue point, the gravitational mass of the quark star is smaller than the one of the initial hadronic star and it is thus energetically convenient to convert the hadronic star. Notice that, in this case, the final quark star configuration has a smaller radius than the one of the hadronic star. Stellar hadronic configurations below the red point cannot convert because they are composed only by nucleons and deltas, for those cases the nucleation time would be infinite. On the other hand, above the red point, all hadronic stars are metastable 
including the maximum mass configuration indicated in the right panel by the red point. Also in this case, on the quark stars branch the star with the same number of baryons has a smaller gravitational mass and the conversion occurs. At variance with the previous case, the final quark star configuration has a radius larger than the one of the hadronic star progenitor.

The process of conversion of a hadronic star into a quark star has been considered in many works, see for instance [40, 47-52]. Remarkably, the velocity of conversion is so high that in many studies, including detailed three-dimensional numerical simulations [52], almost the whole hadronic star is converted within a few milliseconds. Moreover, since the energy released in such a process is of the order of $10^{52} \mathrm{erg}$, it is clear that a similar event would provide very strong neutrino and electromagnetic signals, possibly connected with supernovae and gamma-ray-bursts [40, 42, 53-55]. An interesting outcome of Refs.[40, 49, 52], is that the hydrodynamical combustion stops before the star is completely converted and in some cases few tenths of the initial mass remain unburnt. The subsequent conversion, driven most likely by neutrons and strange quarks diffusion across the interface separating the two phases, releases the rest of the combustion energy on a longer time scale. A detailed study on this possibility is in progress.

Within the scenario above discussed based on the coexistence of hadronic stars and quark stars a very important issue must be addressed: there is no clear evidence of strangelets in cosmic rays. On the other hand, if quark stars do exist it is conceivable that quark stars mergers could occur which then would eject strange quark matter nuggets, similarly to the heavy nuclei which are probably formed during these processes. These strangelets would also possibly convert all neutron stars into quark stars and therefore only one family would actually exist [56]. The process of fragmentation of strange quark matter is largely unknown due to the many uncertainties on the quark matter equation of state, it is possible that even if strange quark matter is ejected, a negligible amount of matter fragments into strangelets [57]. Another possibility has been put forward in [58] where for the first time, strange stars merger simulations have been performed. For values of the total mass of the binary system larger than about $3 M_{\odot}$ a prompt collapse to a black hole is obtained, without a preceding formation of an accretion disk around the black hole. In such cases the mass ejected could be vanishing. In our scenario of two coexisting families of compact stars, quark stars are high mass stars, and therefore the strangelet pollution problem could possibly be solved if prompt collapses occur in their mergers.

\section{Conclusions}

We have considered the problem of formation of baryons heavier than the nucleon in neutron star matter. While a huge literature is available for what concerns hyperons, little has be done regarding the formation of delta isobars. We have shown that the formation of these particles in beta-stable matter is strongly correlated with the density dependence of the symmetry energy, and in particular on the value of the $L$ parameter. The small values of $L$ indicated by recent analyses imply also small critical densities for the formation of deltas. Besides the hyperon puzzle also a delta isobars puzzle could exist which stems from the impossibility of reaching the $2 M_{\odot}$ limit when these baryons are included in the equation of state. On the other hand, while not so massive, those hadronic stars could be quite compact, with radii below $\sim 10.5 \mathrm{~km}$. Such compact configurations would agree with recent analyses of quiescent low mass X-ray binaries although caution is needed because of the many uncertainties in extracting the radii of compact stars. What is then the composition of massive compact stars? We propose that those stellar objects are quark stars. The two families of compact stars, light and compact hadronic stars and large and heavy quark stars, are connected by conversion processes between the two type of stars which occur if hyperons are present in the hadronic star progenitor. Our proposal could be strengthened if even more massive compact stars will be discovered in the future 


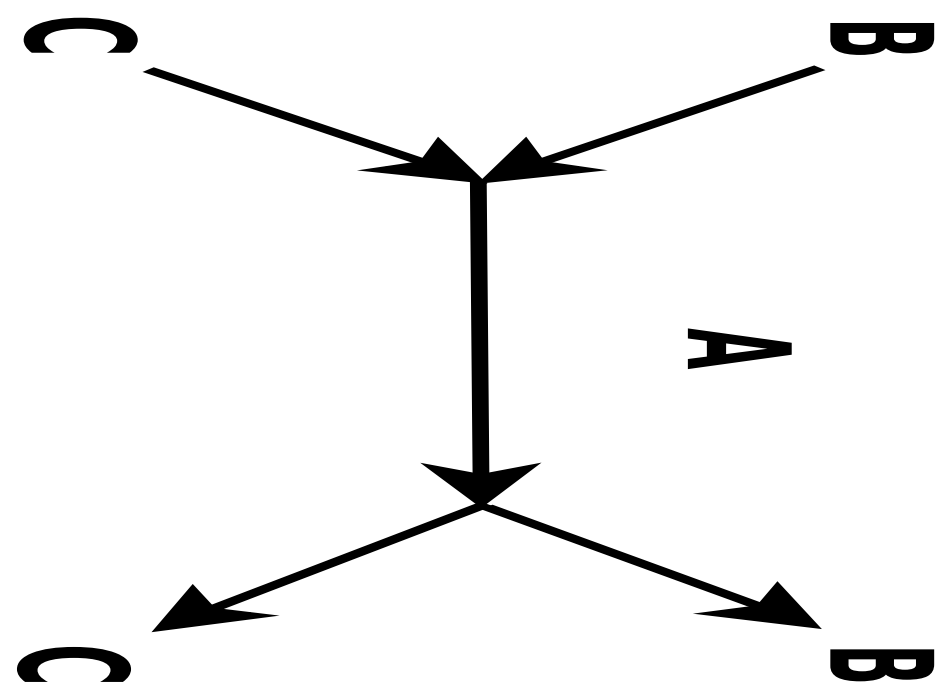

Figure 3. Gravitational mass and baryonic mass as functions of the radius for hadronic stars with and without deltas and for quark stars. The horizontal and vertical lines allow to compare the gravitational mass of hadronic and quark stars at a fixed value of baryonic mass. The green arrows begin with the hadronic star corresponding to the appearance of hyperons (left panel) and the maximum mass and end at the corresponding quark star configuration. Notice that while in the first case the final configuration has a radius smaller than the initial hadronic configuration, the opposite is realised in the second case.

but the crucial measurement would be a precise determination of the radius of a compact star. Small radii, if confirmed, imply a soft equation of state which in turn leads to the formation of hyperons and deltas and a sizable reduction of the maximum mass. On the other hand, if the canonical $1.4 M_{\odot}$ has a radius larger than about $14 \mathrm{~km}$, it implies that the hadronic equation of state is stiff at large densities and even the maximum mass configuration could be composed by just nucleon because its central density is smaller than the thresholds of hyperons and deltas. A phase transition to quark matter could still occur at large densities, as proposed in [59], leading to a third family branch of hybrid stars with masses close to $2 M_{\odot}$. Testing this scenario, which is very much different with respect to ours, would require precise measurements of the radii of the most massive stars.

G.P. acknowledges financial support from the Italian Ministry of Research through the program "Rita Levi Montalcini".

\section{References}

[1] M. Baldo, G. F. Burgio, H.-J. Schulze and G. Taranto, Phys. Rev. C 89 (2014) 048801 [arXiv:1404.7031 [nucl-th]].

[2] W. C. Chen and J. Piekarewicz, arXiv:1408.4159 [nucl-th].

[3] D. Lonardoni, F. Pederiva and S. Gandolfi, Phys. Rev. C 89 (2014) 1, 014314 [arXiv:1312.3844 [nucl-th]].

[4] T. Hell and W. Weise, arXiv:1402.4098 [nucl-th]. 
[5] T. Krüger, I. Tews, K. Hebeler and A. Schwenk, Phys. Rev. C 88 (2013) 025802 [arXiv:1304.2212 [nucl-th]].

[6] P. Demorest, T. Pennucci, S. Ransom, M. Roberts and J. Hessels, Nature 467 (2010) 1081 [arXiv:1010.5788 [astro-ph.HE]].

[7] J. Antoniadis, P. C. C. Freire, N. Wex, T. M. Tauris, R. S. Lynch, M. H. van Kerkwijk, M. Kramer and C. Bassa et al., Science 340 (2013) 6131 [arXiv:1304.6875 [astro-ph.HE]].

[8] S. Weissenborn, D. Chatterjee and J. Schaffner-Bielich, Phys. Rev. C 85 (2012) 065802 [arXiv:1112.0234 [astro-ph.HE]].

[9] M. Baldo, G. F. Burgio and H. J. Schulze, Phys. Rev. C 61 (2000) 055801 [nucl-th/9912066].

[10] I. Vidana, D. Logoteta, C. Providencia, A. Polls and I. Bombaci, Europhys. Lett. 94 (2011) 11002 [arXiv:1006.5660 [nucl-th]].

[11] H. Djapo, B. J. Schaefer and J. Wambach, Phys. Rev. C 81 (2010) 035803 [arXiv:0811.2939 [nucl-th]].

[12] D. Lonardoni, A. Lovato, S. Gandolfi and F. Pederiva, arXiv:1407.4448 [nucl-th].

[13] M. H. van Kerkwijk, R. Breton and S. R. Kulkarni, Astrophys. J. 728 (2011) 95 [arXiv:1009.5427 [astro-ph.HE]].

[14] S. Guillot, M. Servillat, N. A. Webb and R. E. Rutledge, arXiv:1302.0023 [astro-ph.HE].

[15] S. Guillot and R. E. Rutledge, arXiv:1409.4306 [astro-ph.HE].

[16] J. M. Lattimer and A. W. Steiner, Astrophys. J. 784 (2014) 123 [arXiv:1305.3242 [astro-ph.HE]].

[17] S. Bogdanov, Astrophys. J. 762 (2013) 96 [arXiv:1211.6113 [astro-ph.HE]].

[18] K. Hebeler, J. M. Lattimer, C. J. Pethick and A. Schwenk, Astrophys. J. 773 (2013) 11 [arXiv:1303.4662 [astro-ph.SR]].

[19] N. K. Glendenning, Astrophys. J. 293 (1985) 470.

[20] H. Huber, F. Weber, M. K. Weigel and C. Schaab, Int. J. Mod. Phys. E 7 (1998) 301 [nuclth/9711025].

[21] J. M. Lattimer and Y. Lim, Astrophys. J. 771 (2013) 51 [arXiv:1203.4286 [nucl-th]].

[22] A. Drago, A. Lavagno, G. Pagliara and D. Pigato, arXiv:1407.2843 [astro-ph.SR].

[23] A. W. Steiner, M. Hempel and T. Fischer, Astrophys. J. 774 (2013) 17 [arXiv:1207.2184 [astroph.SR]].

[24] K. Wehrberger, C. Bedau and F. Beck, Nucl. Phys. A 504 (1989) 797.

[25] X. m. Jin, Phys. Rev. C 51 (1995) 2260 [hep-ph/9411265].

[26] E. Oset and L. L. Salcedo, Nucl. Phys. A 468 (1987) 631.

[27] J. H. Koch and N. Ohtsuka, Nucl. Phys. A 435 (1985) 765.

[28] J. S. O’Connell and R. M. Sealock, Phys. Rev. C 42 (1990) 2290.

[29] W. M. Alberico, G. Gervino and A. Lavagno, Phys. Lett. B 321 (1994) 177.

[30] Y. Horikawa, M. Thies and F. Lenz, Nucl. Phys. A 345 (1980) 386.

[31] N. K. Glendenning and S. A. Moszkowski, Phys. Rev. Lett. 67 (1991) 2414.

[32] S. Typel, G. Ropke, T. Klahn, D. Blaschke and H. H. Wolter, Phys. Rev. C 81 (2010) 015803 [arXiv:0908.2344 [nucl-th]].

[33] A. Drago, A. Lavagno and G. Pagliara, Phys. Rev. D 89 (2014) 043014.

[34] T. Schurhoff, S. Schramm and V. Dexheimer, Astrophys. J. 724 (2010) L74 [arXiv:1008.0957 [astro-ph.SR]].

[35] E. S. Fraga, R. D. Pisarski and J. Schaffner-Bielich, Phys. Rev. D 63 (2001) 121702 [hep$\mathrm{ph} / 0101143]$. 
[36] M. Alford, D. Blaschke, A. Drago, T. Klahn, G. Pagliara and J. Schaffner-Bielich, Nature 445 (2007) E7 [astro-ph/0606524].

[37] A. Kurkela, P. Romatschke and A. Vuorinen, Phys. Rev. D 81 (2010) 105021 [arXiv:0912.1856 [hep-ph]].

[38] S. Weissenborn, I. Sagert, G. Pagliara, M. Hempel and J. Schaffner-Bielich, Astrophys. J. 740 (2011) L14.

[39] E. S. Fraga, A. Kurkela and A. Vuorinen, The Astrophysical Journal Letters, Volume 781, Issue 2, article id. L25, 5 pp. (2014) [arXiv:1311.5154 [nucl-th]].

[40] G. Pagliara, M. Herzog and F. K. Röpke, Phys. Rev. D 87 (2013) 10, 103007.

[41] K. Iida and K. Sato, Phys. Rev. C 58 (1998) 2538 [nucl-th/9808056].

[42] A. Drago, A. Lavagno and G. Pagliara, Phys. Rev. D 69 (2004) 057505 [nucl-th/0401052].

[43] I. Bombaci, I. Parenti and I. Vidana, Astrophys. J. 614 (2004) 314 [astro-ph/0402404].

[44] I. Bombaci, G. Lugones and I. Vidana, Astron. Astrophys. 462 (2007) 1017 [astro-ph/0603644].

[45] B. W. Mintz, E. S. Fraga, G. Pagliara and J. Schaffner-Bielich, Phys. Rev. D 81 (2010) 123012 [arXiv:0910.3927 [hep-ph]].

[46] D. Logoteta, I. Bombaci, C. Providencia and I. Vidana, Phys. Rev. D 85 (2012) 023003 [arXiv:1203.4159 [nucl-th]].

[47] A. V. Olinto, Phys. Lett. B 192 (1987) 71.

[48] G. Lugones, O. G. Benvenuto and H. Vucetich, Phys. Rev. D 50 (1994) 6100.

[49] A. Drago, A. Lavagno and I. Parenti, Astrophys. J. 659 (2007) 1519 [astro-ph/0512652].

[50] B. Niebergal, R. Ouyed and P. Jaikumar, Phys. Rev. C 82 (2010) 062801 [arXiv:1008.4806 [nucl-th]].

[51] J. E. Horvath, arXiv:1005.4302 [astro-ph.HE].

[52] M. Herzog and F. K. Ropke, Phys. Rev. D 84 (2011) 083002.

[53] A. Drago, G. Pagliara, G. Pagliaroli, F. L. Villante and F. Vissani, AIP Conf. Proc. 1056 (2008) 256 [arXiv:0809.0518 [astro-ph]].

[54] I. Sagert, T. Fischer, M. Hempel, G. Pagliara, J. Schaffner-Bielich, A. Mezzacappa, F.-K. Thielemann and M. Liebendorfer, Phys. Rev. Lett. 102 (2009) 081101 [arXiv:0809.4225 [astro-ph]].

[55] T. Fischer, I. Sagert, G. Pagliara, M. Hempel, J. Schaffner-Bielich, T. Rauscher, F. K. Thielemann and R. Kappeli et al., Astrophys. J. Suppl. 194 (2011) 39 [arXiv:1011.3409 [astro-ph.HE]].

[56] J. L. Friedman and R. R. Caldwell, Phys. Lett. B 264 (1991) 143.

[57] L. Paulucci and J. E. Horvath, Phys. Lett. B 733 (2014) 164 [arXiv:1405.1777 [astro-ph.HE]].

[58] A. Bauswein, H.-T. Janka, R. Oechslin, G. Pagliara, I. Sagert, J. Schaffner-Bielich, M. M. Hohle and R. Neuhauser, Phys. Rev. Lett. 103 (2009) 011101 [arXiv:0812.4248 [astro-ph]].

[59] D. Blaschke, D. E. Alvarez-Castillo and S. Benic, PoS CPOD 2013 (2013) 063 [arXiv:1310.3803 [nucl-th]]. 